Portland State University

PDXScholar

Electrical and Computer Engineering Faculty

Publications and Presentations

$10-1-2001$

Gaussian Beams in Hollow Metal Waveguides:

Experiment

Marius Ghita

Lee W. Casperson

Portland State University

Follow this and additional works at: https://pdxscholar.library.pdx.edu/ece_fac

Part of the Electrical and Computer Engineering Commons

Let us know how access to this document benefits you.

Citation Details

Marius Ghita and Lee W. Casperson, "Gaussian Beams in Hollow Metal Waveguides: Experiment," Appl. Opt. 40, 5459-5462 (2001).

This Article is brought to you for free and open access. It has been accepted for inclusion in Electrical and Computer Engineering Faculty Publications and Presentations by an authorized administrator of PDXScholar. Please contact us if we can make this document more accessible: pdxscholar@pdx.edu. 


\title{
Gaussian beams in hollow metal waveguides: experiment
}

\author{
Marius Ghita and Lee W. Casperson
}

\begin{abstract}
Gaussian beams have been widely used for propagating electromagnetic waves in free space and in certain other optical systems. It has been suggested that recurring forms of such beams might also be useful for propagation in planar or rectangular metal waveguides. Experimental verification of the recurrence of the Gaussian field distribution in metal waveguides is reported here. (C) 2001 Optical Society of America

OCIS codes: $230.0230,230.7370,230.7390,350.5500$.
\end{abstract}

\section{Introduction}

Gaussian beams have long been used to describe electromagnetic waves propagating in media that have at most quadratic variations of the index of refraction and of the gain or loss in the vicinity of the beam. ${ }^{1,2}$ At every plane along the beam propagation path the amplitude distribution remains Gaussian, whereas the phase fronts remain spherical. More-complicated off-axis polynomial Gaussian beams have also been studied, and their propagation in a range of complex and misaligned systems is now known. ${ }^{3}$ Similar properties have been found to be shared by general families of Bessel-Gaussian, exponential Gaussian, and trigonometric Gaussian beams. ${ }^{4}$ However, there are also waveguiding structures for which the field distribution fluctuates periodically with propagation distance. Waveguides of this type have been considered for a variety of acoustic ${ }^{5,6}$ and optical wave fields. ${ }^{7-21}$ Because of this periodic property, these fields have been referred to as recurring beams. Such beams are of particular interest for practical applications involving the propagation of electromagnetic fields in hollow metal waveguides.

With lower-frequency electromagnetic fields the transverse beam dimensions and diffraction angles may become too large for useful unguided propagation, and more-confining waveguiding systems must be employed. When suitable dielectric media are

M. Ghita and L. W. Casperson (lcaspers@ee.pdx.edu) are with the Department of Electrical and Computer Engineering and Department of Physics, Portland State University, Portland, Oregon 97207-0751.

Received 1 February 2001; revised manuscript received 1 February 2001.

0003-6935/01/305459-04\$15.00/0

(C) 2001 Optical Society of America not available, hollow metal waveguides may be a good option. These guides are able to provide lowloss transmission of electromagnetic wave fields even when the wavelength is not much less than the transverse dimensions of the guide, and they are also effective with short wavelengths and high power levels. However, the familiar sinusoidal (rectangular geometry) or Bessel (circular geometry) waveguide modes are not without disadvantages, especially for optical frequency applications. In optical frequency systems it is often necessary to couple free-space propagating beams into or out of waveguides. Most applications of waveguide laser amplifiers and oscillators require such beams, and thus transformations between waveguide and beam field configurations are frequently needed. Such transformations never lead to the ideal field distributions that one might hope for, and they also may involve significant power loss. It is in this context that one should consider the possibility of using recurring beams that have more-useful transverse profiles at certain discrete planes along the propagation path. Beams that have Gaussian amplitude distributions are of particular interest, and it has been shown that recurring Gaussian beams can describe wave propagation in hollow metal waveguides. This demonstration has been based on a Green function integration method $^{17-20}$ and on the standard Gaussian beam propagation formulas for a region with reflecting boundaries. $^{21}$ Similar results are also sometimes applicable to dielectric waveguides.

In spite of the potential importance of the recurring propagation of Gaussian beams in hollow metal waveguides, we know of no direct experimental verification of the theoretical predictions. The main experimental results have involved recurrence of non-Gaussian aperture fields in dielectric rather than hollow metal waveguides. In this paper we 


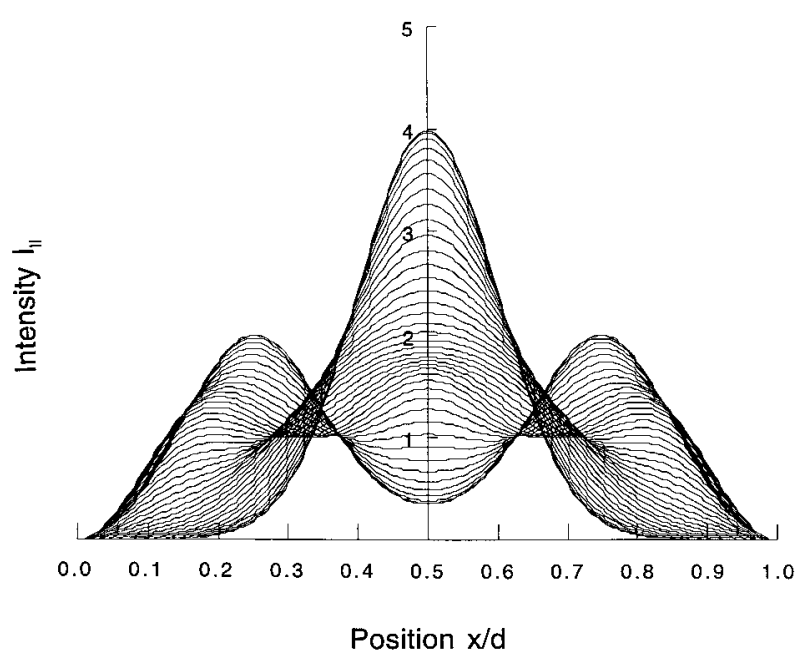

Fig. 1. Series of transverse intensity profiles of a normalized on-axis Gaussian beam interacting with flat waveguide surfaces located at position $x= \pm 0.5 d$, where $d$ is the distance between the waveguide surfaces. The beam is polarized parallel to the surfaces, the input waist spot size is $w_{0}=0.2 d$, and the propagation distance between successive profiles is $z=0.1 z_{0}$ (ten plots per Rayleigh length). The original near-Gaussian profile recurs after approximately eight Rayleigh lengths.

demonstrate experimentally that good fidelity recurrence of the Gaussian beam produced by a simple spherical mirror gas laser resonator can readily be achieved in a waveguide consisting of two metallized front surface mirrors.

A brief summary of the equations that govern Gaussian beam recurrence is included in Section 2, and experimental confirmation of these results is reported in Section 3.

\section{Formulas}

A Gaussian beam will always experience severe distortions of its amplitude and phase profiles when it is propagating through a hollow metal waveguide. For certain waveguide geometries, however, the beam will become Gaussian again at specific locations along the waveguide. This is true whether the beam enters the waveguide on center or off center and whether the beam is polarized parallel or perpendicular to the waveguide surfaces. ${ }^{21}$

In our experiment a Gaussian beam enters a parallel-plate waveguide with a waist at the center point of the waveguide and with the propagation direction along the waveguide axis. To obtain the shortest recurrence distance, we polarize the beam field parallel to the waveguide surfaces. Figure 1 is a theoretical plot of a series of transverse intensity profiles for a Gaussian beam that is undergoing diffraction and reflection from the flat waveguide surfaces. The waist spot size $w_{0}$ in this example is 0.2 times the waveguide wall spacing, and the propagation distance between successive profiles is 0.1 times the Rayleigh length:

$$
z_{0}=\pi w_{0}^{2} / \lambda
$$

where $\lambda$ is the wavelength of the beam. The intensity is seen to spread into two symmetrical peaks and then return to its initial near-Gaussian profile after approximately eight Rayleigh lengths. As required by the boundary conditions for this polarization, the intensity always remains zero at the reflecting surfaces.

For the configuration just described, the Gaussian beam recurs in the distance

$$
z_{\|}=\frac{z_{0}}{\pi\left(w_{0} / d\right)^{2}},
$$

where $d$ is the spacing between the waveguide walls. ${ }^{21}$ When it is combined with the Rayleigh length given in Eq. (1), this recurrence distance can also be written simply as

$$
z_{\|}=d^{2} / \lambda \text {. }
$$

If this distance coincides with the length of the waveguide, the beam will emerge from the waveguide with the same Gaussian profile with which it entered.

One can obtain the narrow input beam that is appropriate for the waveguide by focusing a laser beam with a lens. The lens would typically be positioned such that the waist of the beam emerging from it would occur at the entrance of the waveguide. The distance of the waist from the lens can be found by a standard beam matrix calculation. If the beam incident upon the lens has flat phase fronts, this distance is

$$
z=\frac{f}{1+\left(f / z_{1}\right)^{2}}
$$

where $f$ is the focal length of the lens and $z_{1}=\pi w_{1}{ }^{2} / \lambda$ is the Rayleigh length of the beam incident upon the lens. One finds further that at this distance the beam has the waist spot size

$$
w_{0}=\frac{f \lambda}{\pi w_{1}\left[1+\left(f / z_{1}\right)^{2}\right]^{1 / 2}} .
$$

In a typical configuration the Rayleigh length $z_{1}$ of the beam incident upon the lens will be much larger than the focal length of the lens. Thus Eq. (4) will reduce to the simpler result that the waveguide is removed from the lens by a distance equal to the focal length

$$
z=f
$$

and from Eq. (5) the spot size of the beam at the entrance of the waveguide is approximately

$$
w_{0}=\frac{f \lambda}{\pi w_{1}} .
$$

These basic formulas are all that are required for designing an experiment to demonstrate the recurrence property of a Gaussian input beam in a planar waveguide. 


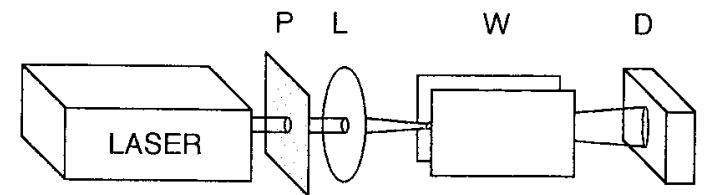

Fig. 2. Schematic representation of the experimental setup for demonstrating the recurrence of a Gaussian beam in a planar metal waveguide. The beam from a helium-neon laser is polarized by polarizer $\mathrm{P}$, focused by lens $\mathrm{L}$, transmitted through waveguide $\mathrm{W}$, and propagated to detector array $\mathrm{D}$.

\section{Experiment}

The light source used in our experiments is an ordinary low-power unpolarized helium-neon laser with a wavelength of $0.633 \mu \mathrm{m}$ and a $\mathrm{TEM}_{00}$ Gaussian output beam with a spot size of $\sim 1.0 \mathrm{~mm}$. All intensity distributions are characterized with a beam profiler that uses a CCD camera. The overall experimental setup is sketched in Fig. 2. A linearly polarized beam is needed for our experiments, so the output beam of the laser is first passed through a linear polarizer, as shown in Fig. 2. A lens with a $100-\mathrm{mm}$ focal length is used to narrow the beam such that it is not clipped at the entrance of the waveguide. The guide is composed of two front surface mirrors, parallel to each other. The location of the lens and the orientation of the mirrors can be adjusted individually with micrometer translation stages to allow for precise positioning.

From Eq. (7), the spot size of the focused Gaussian beam is $\sim w_{0}=0.02 \mathrm{~mm}$, and this dimension should be substantially less than the spacing of the waveguide surfaces. If the distance given in Eq. (3) coincides with the length of the waveguide, the beam will recover its original Gaussian form at its exit from

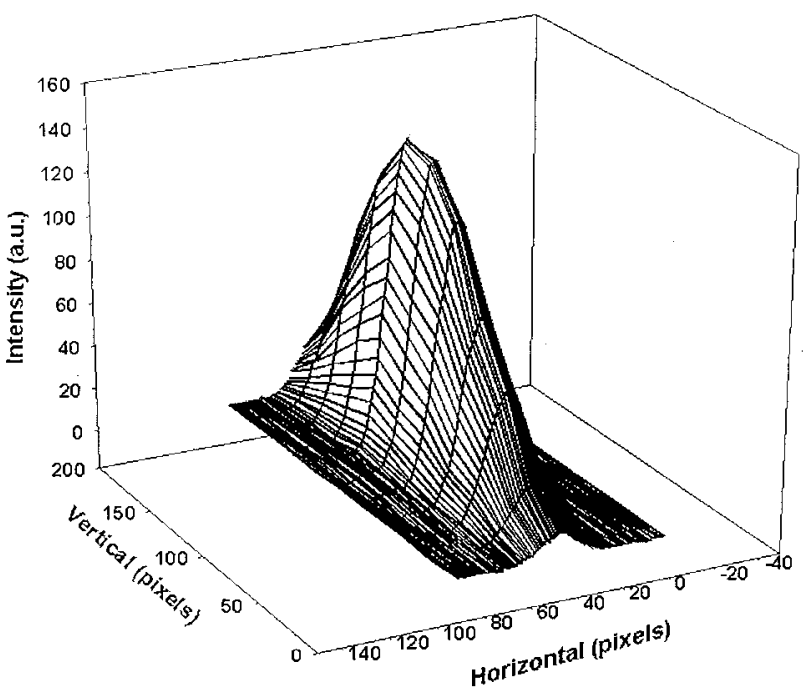

Fig. 3. Intensity profile of the elliptical Gaussian beam incident upon a detector array after transmission through a planar waveguide. The elliptical shape results from the greater diffraction of the initially symmetric Gaussian beam in the direction parallel to the waveguide surfaces.

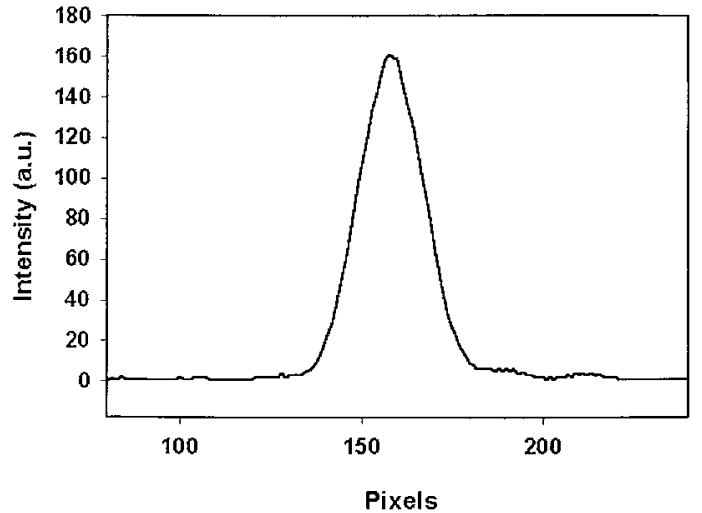

Fig. 4. Intensity profile of the detected beam in the direction perpendicular to the waveguide surfaces.

the guide. The mirrors that constitute the waveguide in our experiments have a length in the propagation direction of $160 \mathrm{~mm}$. As this length could not easily be changed, the spacing of the mirrors had to be adjusted to ensure that $z_{\|}$in Eq. (3) would equal the waveguide length. In this way the ideal spacing for our experiment was found to be $\sim 0.32 \mathrm{~mm}$, in agreement with measurements.

Figure 3 shows the experimental intensity profile of the beam after it has left the waveguide and arrived at the detector array. In a direction parallel to the waveguide surfaces (vertical) the beam is not restricted by the waveguide. Thus the beam can diverge freely in the vertical direction, starting at the beginning of the waveguide. However, in a direction perpendicular to the waveguide surface, the beam at the output has the same profile as at the input. Thus the beam arriving at the detector has diffracted in the vertical direction with the same diffraction angle but over a longer distance than it has diffracted in the horizontal direction. This is the reason that the profile in Fig. 3 has an elliptical Gaussian shape.

Figure 4 shows in more detail the intensity profile of the beam in the direction perpendicular to the waveguide surfaces after the beam has exited from the waveguide. This profile fits closely a Gaussian curve, and it is not significantly different except in scale from the profile that is obtained without the presence of a guide.

\section{Conclusion}

On the one hand, Gaussian beams have wellestablished advantages for the propagation of electromagnetic fields in free space and in certain lenslike media. On the other hand, for some wavelengths and power levels it has proved more efficient or economical to propagate electromagnetic fields in hollow metal waveguides. ${ }^{21}$ However, it would usually be supposed that Gaussian beams and metal waveguides are incompatible. In this study it has been shown experimentally that field solutions for metal waveguides in the form of recurring Gaussian beams can be readily obtained. With these results it becomes possible and desirable to consider simple 
hollow metal waveguides among the optical elements that are capable of low-loss transmission of Gaussian beams.

The authors are pleased to acknowledge valuable correspondence from E. F. Kuester.

\section{References}

1. H. Kogelnik, "On the propagation of Gaussian beams of light through lenslike media including those with a loss or gain variation," Appl. Opt. 4, 1562-1569 (1965).

2. L. W. Casperson, "Gaussian light beams in inhomogeneous media," Appl. Opt. 12, 2434-2441 (1973).

3. A. A. Tovar and L. W. Casperson, "Generalized beam matrices: Gaussian beam propagation in misaligned complex optical systems," J. Opt. Soc. Am. A 12, 1522-1533 (1995), and references therein.

4. See, for example, L. W. Casperson, D. G. Hall, and A. A. Tovar, "Sinusoidal-Gaussian beams in complex optical systems," J. Opt. Soc. Am. A 14, 3341-3348 (1997), and references therein.

5. D. E. Weston, "A moiré fringe analog of sound propagation in shallow water," J. Acoust. Soc. Am. 32, 647-654 (1960).

6. D. E. Weston, "Sound focusing and beaming in the interference field due to several shallow-water modes," J. Acoust. Soc. Am. 44, 1706-1712 (1968).

7. L. A. Rivlin and V. S. Shul'dyaev, "Multimode waveguides for coherent light," Radiophys. Quantum Electron. 11, 318-321 (1968).

8. O. Bryngdahl, "Image formation using self-imaging techniques,” J. Opt. Soc. Am. 63, 416-419 (1973).
9. R. Ulrich, "Light-propagation and imaging in planar optical waveguides," Nouv. Rev. Opt. 6, 253-262 (1975).

10. R. Ulrich, "Image formation by phase coincidences in optical waveguides," Opt. Commun. 13, 259-264 (1975).

11. R. Ulrich and G. Ankele, "Self-imaging in homogeneous planar optical waveguides," Appl. Phys. Lett. 27, 337-339 (1975).

12. A. Simon and R. Ulrich, "Fiber-optical interferometer," Appl. Phys. Lett. 31, 77-79 (1977).

13. O. Bryngdahl, "On light distribution in optical waveguides," J. Opt. Soc. Am. 68, 310-315 (1978).

14. R. Ulrich and T. Kamiya, "Resolution of self-images in planar optical waveguides," J. Opt. Soc. Am. 68, 583-592 (1978).

15. J. C. Campbell and T. Li, "Electro-optic multimode waveguide switch," Appl. Phys. Lett. 33, 710-712 (1978).

16. E. E. Grigor'eva and A. T. Semenov, "Waveguide image transmission in coherent light (review)," Sov. J. Quantum Electron. 8, 1063-1073 (1978).

17. D. C. Chang and E. F. Kuester, "A hybrid method for paraxial beam propagation in multimode optical waveguides," IEEE Trans. Microwave Theory Tech. MTT-29, 923-933 (1981).

18. E. F. Kuester, G. S. Dow, and D. C. Chang, "Coupling and imaging of Gaussian beams in parallel dielectric slab waveguides," Arch. Elektr. Uebertrag. 36, 417-435 (1982).

19. E. F. Kuester and D. C. Chang, "Imaging and propagation of beams in metallic or dielectric waveguides," in Hybrid Formulation of Wave Propagation and Scattering, L. B. Felsen, ed. (Nijhoff, Boston, Mass., 1984), pp. 185-194.

20. A. R. Mahnad and E. F. Kuester, "Image formation in circular waveguides and optical fibers," IEEE MTT-S International Microwave Symposium Digest, 122-124 (1983).

21. L. W. Casperson, "Gaussian beams in hollow metal waveguides,” J. Opt. Soc. Am. A 17, 1115-1123 (2000). 